\title{
WestVirginiaUniversity
}

THE RESEARCH REPOSITORY @ WVU

Graduate Theses, Dissertations, and Problem Reports

2011

\section{Clinician Detection of Depression in Late Life}

Jeffrey J. Gregg

West Virginia University

Follow this and additional works at: https://researchrepository.wvu.edu/etd

\section{Recommended Citation}

Gregg, Jeffrey J., "Clinician Detection of Depression in Late Life" (2011). Graduate Theses, Dissertations, and Problem Reports. 3314.

https://researchrepository.wvu.edu/etd/3314

This Thesis is protected by copyright and/or related rights. It has been brought to you by the The Research Repository @ WVU with permission from the rights-holder(s). You are free to use this Thesis in any way that is permitted by the copyright and related rights legislation that applies to your use. For other uses you must obtain permission from the rights-holder(s) directly, unless additional rights are indicated by a Creative Commons license in the record and/ or on the work itself. This Thesis has been accepted for inclusion in WVU Graduate Theses, Dissertations, and Problem Reports collection by an authorized administrator of The Research Repository @ WVU. For more information, please contact researchrepository@mail.wvu.edu. 


\title{
Clinician Detection of Depression in Late Life
}

\author{
Jeffrey J. Gregg
}

\author{
Thesis submitted to the Eberly College of Arts and Sciences \\ at West Virginia University \\ in Partial Fulfillment of the Requirements for the degree of \\ Master of Science \\ In \\ Psychology
}

\author{
Amy Fiske, Ph. D., CBSM \\ Barry Edelstein, Ph.D. \\ Aaron Metzger, Ph.D.
}

Department of Psychology

\begin{abstract}
Morgantown, WV
\end{abstract}
2011

Keywords: Late-life depression, Diagnosis, Depressed affect, Cognitive Impairment 


\section{ABSTRACT \\ Clinician Detection of Depression in Late Life}

\section{Jeffrey J. Gregg}

Depression in late life is associated with decreased cognitive functioning, increased social and functional impairment, and overall increased mortality. Because of these negative health implications, the accurate assessment of late-life depression is a critical public health issue. Prior research has consistently demonstrated that older adults typically seek and receive mental health services from general medical settings instead of mental health specialty clinics. Unfortunately, many studies have shown that primary care physicians often fail to recognize depression. In addition, older adults are less likely than younger adults to endorse symptoms of sadness or dysphoria. Those with cognitive impairment are also less likely to exhibit depressed affect and it is often difficult to distinguish between depression and cognitive impairment. The present study examined the possible moderating effect of level of cognitive functioning and the endorsement of depressed affect on the relation between overall level of depressive symptoms and the clinician diagnosis of depression in a population-based sample of 344 adults (age $\geq 50$ ). Results indicated that the endorsement of one item that assessed depressed affect on the Center for Epidemiologic Studies- Depression scale (i.e., "I felt depressed") moderated the relation between overall level of depressive symptoms and the clinician diagnosis of late-life depression.

However, a separate item that assessed depressed affect on the same scale (i.e., "I felt sad") did not moderate this relation. In addition, level of cognitive functioning did not moderate the relation between overall level of depressive symptoms and the clinician diagnosis of depression in late-life. Implications, limitations, and future directions are discussed. 


\section{Table of Contents}

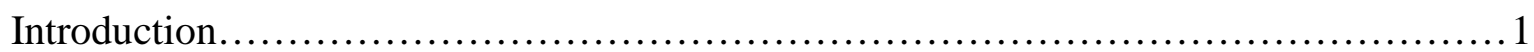

Method......................................................................

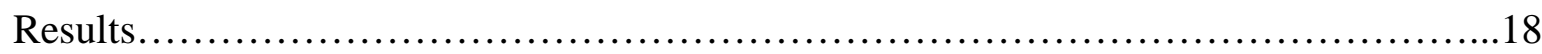

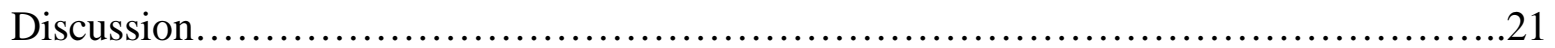

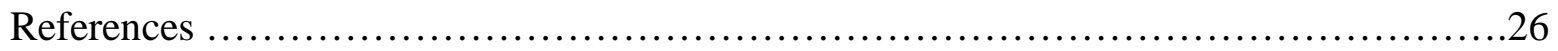

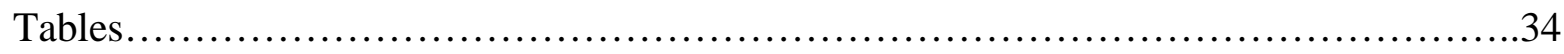




\section{Clinician Detection of Depression in Late Life}

Major depression has been shown to be less prevalent in older adults than in younger adults (Hasin, Goodwin, Stinson, \& Grant, 2005). However, 15\% of community dwelling older adults are estimated to have clinically significant depressive symptoms. Though major depression appears to be less common in older adults, it is associated with decreased cognitive functioning, increased social and functional impairment, and overall increased mortality (Blazer, 2003). In addition, depression is one of the most prominent risk factors for suicide in late life and $85 \%$ of older adults who commit suicide are depressed (Conwell \& Brent, 1996). Because of the negative health implications associated with depression in late life, the accurate assessment of depression in older adults is a critical public health issue.

Prevalence rates for major depression in community dwelling adults aged 65 and older range between 1\% and 5\% (Hasin et al., 2005). Depression rates for special populations of older adults tend to be higher, including medical inpatients (10\%-15\%) and residents of long-term care facilities (14\%-42\%; Blazer, 2003; Djernes, 2006). Though prevalence rates of major depression represent a significant decline from middle age to late life, endorsement of depressive symptoms actually increases with age (Newmann, 1989). These statistics suggest that the detection of latelife depression is a complex issue. Furthermore, many studies have shown that major depression is underdiagnosed in primary care, where most older adults seek treatment (e.g., Katon \& Schulberg, 1992). The current study seeks to characterize factors that may interfere with the detection of depression in late life, particularly, the differential presentation of depression in late life and the presence of cognitive impairment in older adults. 
Prior research has consistently demonstrated that older adults typically seek and receive mental health services from general medical settings (i.e. primary care physicians) instead of mental health specialty clinics (Harmon, Veazie, \& Lyness, 2006). One recent study found that older adults were one third as likely as younger adults to seek specialty mental health services (Bogner, de Vries, Maulik, \& Unützer, 2009). Licht-Strunk et al. (2005) examined the prevalence of depression in 5,686 Dutch older adults who consulted their primary care physician. They found that $13.7 \%$ of the participants screened positive for major depression, and $10.2 \%$ screened positive for minor depression. Overall depression increased with age, although incidence of minor depression also increased with age. The study did not report how many individuals were diagnosed with depression by their primary care physician.

Unfortunately, many studies have demonstrated that primary care physicians often fail to recognize depression (Jencks, 1985; Katon \& Schulberg, 1992). Mitchell, Vaze, and Rao (2009) conducted a meta-analysis examining the clinical diagnosis of depression in primary care settings utilizing 50,371 patients across 41 studies. They found that general practitioners correctly identified depression in only $47.3 \%$ of cases. Using the 19 studies that employed both rule-in and rule-out categories, they found that physicians had a weighted sensitivity of only $50.1 \%$, but a specificity of $81.3 \%$. Additionally, Simon and Von Korff (1995) found that only 29\% of patients with subthreshold depression were accurately assessed by their primary care physician. Symptom severity will be discussed in further detail and is an important theme in this proposal.

One possible explanation for low rates of detection of depression in primary care is that many depressed patients who present to their physicians have a high level of medical comorbidity, with their chief complaints being somatic symptoms. The evaluation of these somatic symptoms takes time and resources and may complicate and delay a diagnosis of 
depression (Klinkman, 2003). Adults with certain age-related medical conditions, such as Parkinson’s disease and Alzheimer's disease, are also likely to experience milder forms of depression, and without symptoms of dysphoria (Ehrt, Brønnick, Leentjens, Larsen, \& Aarsland, 2006; Olin et al., 2002). In fact, Olin et al. (2002) have developed and proposed new diagnostic criteria for Depression of Alzheimer's disease, where fewer symptoms are required (three instead of five) and symptoms of irritability and social withdrawal have been added.

A review of barriers to the diagnosis of depression in primary care by Docherty (1997) identifies several other challenges in the detection of depression in primary care, including patient-related, physician-related, and system-related factors. Patient-related factors that were identified include lack of understanding of depression, variability in clinical presentation, and reluctance to endorse depressive symptoms due to the negative stigma associated with mental illness. Physician-related barriers to the detection of depression include lack of knowledge/training in depression, reluctance to inquire about depression, and lack of confidence in treating depression. Important system-related factors that interfere with depression detection in primary care include reimbursement issues, lack of time available to patients, and discontinuity in the physician/patient relationship.

Research has also demonstrated that cases of late-life depression that go undetected in primary care settings have poorer prognosis than those that are detected (Licht-Strunk et al., 2009). Though many studies have found that primary care physicians often fail to recognize depression in older adults and several barriers have been identified, prior research has not adequately examined the possibility that physicians may be more or less likely to detect depression when certain symptom constellations are present. 


\section{Presentation of late-life depression}

Perhaps one of the most salient factors that may interfere with the clinician's ability to detect depression in older adults is the differential presentation of late-life depression. Research has demonstrated that older adults are less likely than younger adults to endorse symptoms of sadness or dysphoria. Gallo, Anthony, and Muthén (1994) examined data collected by the Epidemiologic Catchment Area Program to compare age differences in depression symptom endorsement. The researchers hypothesized that the emphasis placed on the dysphoria symptom by the Diagnostic and Statistical Manual, $3^{\text {rd }}$ ed. (DSM-III) may have led to an underestimation in the prevalence of depression in older adults. The study consisted of 3,191 individuals from thegreater Baltimore area and 3,469 from the Durham-Piedmont (NC) area. Gallo and colleagues compared depressive symptomology between adults over age 65 and adults under age 65 within these samples. Symptoms of depression were assessed using the Major Depression section of the Diagnostic Interview Schedule (DIS), which is based on the criteria for major depression found in the DSM-III. A latent trait analysis revealed that adults aged 65 and older were less likely to endorse dysphoria or anhedonia than younger adults, even after controlling for gender, ethnicity, marital status, employment status, cognitive impairment, and overall level of depression. Additionally, results showed that older adults were more likely to endorse appetite disturbance and thoughts of death than younger adults.

Newmann, Engel, and Jensen (1991) had similar findings in a sample of women aged 50 and older. To examine age differences, the sample was divided into a younger cohort aged 52-65 $(\mathrm{N}=184)$ and an older cohort aged 66 and older $(\mathrm{N}=184)$. Symptoms of depression were assessed using 16 items from the Symptom Checklist-90-Revised (SCL-90-R) Depression and Additional Symptoms Scales. These items are also derived from the DSM-III criteria for Major 
Depression. Results provided evidence for two separate presentations of depression: one termed “depressive syndrome,” in which the primary symptom is dysphoria, and another termed “depletion syndrome,” in which the primary symptoms are appetite loss, anhedonia, thoughts of dying, and hopelessness. Significantly higher levels of depletion syndrome were found in the older cohort, compared to higher levels of depressive syndrome in the younger cohort. These findings suggest that older adults may be more likely to endorse a presentation of depression that differs from a more classic depressive syndrome, where dysphoric symptoms are the most prominent.

These studies have demonstrated that the phenomenon of non-dysphoric depression is more prevalent in older adults than in younger adults. Two different conceptualizations of depression without dysphoria have been proposed. The first, “depletion syndrome” (previously discussed) has the prominent symptoms of anhedonia, loss of appetite, hopelessness, and thoughts of dying (Newmann et al., 1991). Another presentation, termed “depression without sadness,” is characterized by feelings of hopelessness/helplessness, feelings of worthlessness, and thoughts of death or suicide (Gallo, Rabins, Lyketsos, Tien, \& Anthony, 1997). The prominent symptoms of sadness and loss of interest are omitted in “depression without sadness.” Because neither dysphoria nor anhedonia is endorsed, the DSM-IV criteria for major depression cannot be met. However, treatment is still warranted, as individuals with this presentation are at an increased risk for psychological distress, functional impairment, cognitive impairment, and death (Gallo \& Rabins, 1999).

\section{Cognitive Impairment}

One particular area of concern in the assessment of late life depression is cognitive impairment. Specifically, the presentation of depression in individuals with cognitive 
impairment differs from a more classic conceptualization of depression where the chief symptom is depressed mood. Many studies have noted the differential symptom profile of depressed individuals with cognitive impairment. As mentioned previously, individuals with Alzheimer's disease are more likely to experience a sub-syndromal level of depression with chief symptoms of irritability and social withdrawal (Olin et al., 2002). Likewise, stroke victims and individuals with vascular dementia are more likely to display vegetative symptoms (i.e., weight loss, fatigue) than symptoms of dysphoria or sadness (Pardiso et al., 2008; Park et al., 2007).

Another obstacle that may impede the valid assessment of depression in the context of cognitive impairment is distinguishing between the two (Storandt \& Vandenbos, 1994). This is especially difficult because many symptoms overlap, and depression and cognitive impairment can co-exist. Some overlapping symptoms include difficulty concentrating, impaired acquisition of new information, and apathy (American Psychiatric Association, 2000). Dementia is often accompanied by co-morbid psychiatric symptoms such as depression, anxiety, agitation, and psychosis (Lopez et al., 2003). Reports of the occurrence of depressive symptoms in adults with dementia vary widely, but most center between 30-50\% (Steffens \& Potter, 2007). There are several possible explanations for this comorbidity. Depression may result as a reaction to a dementia diagnosis (Jha, Tabet, \& Orrell, 2001) or depression may be a prodromal symptom of dementia (Schweitzer, Tuckwell, O’Brien, \& Ames, 2002). Neuronal loss has also been hypothesized to induce depression in older adults with dementia (Lyness, Zarrow, \& Chui, 2003). The co-occurrence of depression and dementia is of particular clinical concern because it is also associated with adverse health outcomes such as increased mortality (Mehta et al., 2003) and functional impairment (Logsdon, McCurry, \& Teri, 2008). In addition, caregivers of individuals with dementia and co-morbid depression have been shown to have elevated levels of 
distress and depression compared to caregivers of demented individuals without depression (Teri, McKenzie, \& LaFazia, 2005).

Another complication is that most of the commonly used self-report measures for depression ask respondents to recall experiences over a specified period of time (i.e., Beck Depression Inventory, Center for Epidemiologic Studies- Depression Scale). This may be problematic for individuals with cognitive impairment, as they have difficulties with memory. Only one study to date has been conducted aimed at identifying the level of cognitive impairment at which individuals are no longer accurate self-reporters of depression. Bédard et al. (2003) examined responses on the Geriatric Depression Scale (GDS) and found that participants with Mini-Mental State Exam (MMSE) scores of 18 or below (suggestive of moderate-severe dementia) answered significantly fewer items, were more likely to endorse questions framed positively, and demonstrated less consistency in responses between two time periods. However, no studies have replicated these findings or examined other self-report measures for depression.

\section{Possible Moderators of Clinician Detection of Late Life Depression}

There are a number of factors that complicate clinicians’ abilities to detect late-life depression. Several studies have shown that clinicians may be more likely to detect depression when symptoms are more severe. Coyne, Schwenk, and Fechner-Banks (1995) conducted a study to identify factors that facilitated or interfered with the detection of depression in primary care. They examined physician performance in diagnosing depression in 1580 patients screened for major depression using the Center for Epidemiologic Studies- Depression Scale (CES-D) and the Structured Clinical Interview for DSM-III-R (SCID). Coyne and colleagues found that those who were undetected had lower CES-D scores and higher levels of functioning compared to 
patients who were detected by their physician. Additionally, detected patients rated themselves as being more depressed and as having less energy than undetected participants. These results are particularly relevant, since older adults are more likely than other age groups to experience subsyndromal levels of depression (Fiske, Wetherell, \& Gatz, 2009).

The differential presentation of late-life depression may also affect the likelihood of clinician detection. Older adults are more likely than younger adults to present with nondysphoric depression (Gallo et al., 1994; Newmann et al., 1991). In addition, as previously discussed, older adults with cognitive impairment often experience a subsyndromal level of depression with chief symptoms of anhedonia and irritability (Olin et al., 2002). Thus, it is possible that clinicians may be under-diagnosing depression in older adults if they are differentially biased towards diagnosing depression when the symptom of sadness or dysphoria is endorsed.

There are many possible explanations for why clinicians might privilege dysphoria when diagnosing depression. In particular, the DSM-IV criteria for major depression emphasize dysphoria (American Psychiatric Association, 2000), which is less frequently endorsed by older adults (Gallo et al., 1994; Newmann, et al., 1991; Fiske \&O’Riley, 2008). Clinicians may also have difficulty in diagnosing depression because current diagnostic criteria for major depression require a judgment that presenting symptoms cannot be attributed to the effects of a medication, a medical condition, or a recent bereavement (American Psychiatric Association, 2000), all of which increase with age. As medical conditions and bereavement increase, it may become more difficult for clinicians to judge what symptoms are attributable to depression.

A number of recommendations have been proposed to address screening for depression in primary care; though, none takes into account the differential symptom presentation of 
depression in late life. The U.S. Preventive Services Task Force, a panel of experts in primary care and prevention that develops recommendations for clinical preventive services, advocates for screening for depression in primary care settings, even if the screening consists of only two questions regarding depressed mood and anhedonia in the past 2 weeks (U.S. Preventive Services Task Force, 2009). This screening may not be sensitive enough for older adults, since symptoms may be mistakenly attributed to physical illness or bereavement, older adults have higher incidence of milder depression, and depression in late-life often presents without sadness/depressed mood. Primary care physicians often note that the atypical presentation of late-life depression is a barrier to the assessment and treatment of depression in older adults (Gallo, Ryan, \& Ford, 1999).

Little research has been conducted to determine whether clinicians privilege certain symptoms when assessing depression. Klinkman, Coyne, Gallo, \& Schwenk (1998) conducted a study with 50 primary care physicians and 1580 patients in southeastern Michigan to examine clinical characteristics of patients with or without depression who were correctly or incorrectly identified. A weighted sample of 372 patients was administered the Structured Clinical Interview for DSM-III-R (SCID) within a two-week window following a visit to their primary care physician. Immediately following the patient's visit to his/her physician, the physician was asked to rate the level of depression, general health, and stress of the patient. The physician was also asked to report (yes or no) whether the patient had a clinically significant level of depressive symptoms. The weighted sample of patients was then categorized into true positives, false positives, false negatives, and true negatives based on scores on the SCID and identification or non-identification by primary care physician. Results showed that depressed patients were more likely to be detected by their physician if they had previously received treatment for depression, 
rated themselves as having higher levels of depression, and scored higher on the CES-D. There were no differences in the clinical characteristics of the false negative and false positive group. Results suggested that physicians discriminated between these two groups solely based on their knowledge of the patient's psychiatric history.

With regard to somatic symptoms such as fatigue and sleep difficulty, Klinkman (2003) notes that they have little predictive value in primary care settings. He cites that the baseline rates of fatigue, sleep difficulty, and appetite disturbance in non-depressed primary care patients is about $20 \%$. However, there is evidence that not attending to these somatic symptoms may impair the detection of depression in primary care. One recent study examined the effect of patients' symptom presentation on the recognition of depression by physicians in a sample with 191 primary care providers and 1910 patients in Italy (Menchetti, Murri, Bertakis, Bortolotti, \& Berardi, 2009). The researchers found that odds ratios for non-recognition were significantly higher for patients presenting with physical complaints (2.3; 95\% CI=1.1-5.3) and pain (4.1; 95\% CI=1.6-9.9) compared to patients with chief psychological complaints (Menchetti et al., 2009). These findings are particularly relevant to older adults, as they are less likely than younger adults to endorse dyspohoria or sadness, and more likely to endorse the somatic complaints of sleep disturbance and appetite loss (Newmann et al., 1991; Gallo et al., 1994). It should be noted that many medical illnesses are known to cause depression (Alexopoulos et al., 2001) and many clinicians note the importance of considering somatic symptoms, but also encourage caution in interpretation (Edelstein, Drozdick, \& Ciliberti, 2010).

Research has demonstrated that physicians are less likely to detect depression in individuals with chief somatic complaints and more likely to detect depression in individuals with greater symptom severity and with prior clinical history. These studies demonstrate that 
symptom presentation does make a difference in whether a clinician is more or less likely to detect depression. However, more research is needed to determine what symptoms (i.e., depressed mood) may have greater predictive value in the diagnosis of depression.

\section{Statement of the problem}

Depression in late life is associated with many negative health outcomes, including increased risk of suicide, decreased cognitive functioning, increased functional and social impairment, and increased mortality. Many evidence-based treatments for late-life depression exist, even in the context of cognitive impairment and dementia. Though many treatments exist, older adults are less likely than younger adults to be diagnosed with depression and psychotherapy is underutilized in older adults compared to pharmacological treatments (Fiske et al., 2009). Prior research has not adequately examined potential clinician-related factors that may interfere with the detection of depression in late life.

The differential presentation of depression in late life and the presence of cognitive impairment have been identified as potential barriers to the assessment and detection of depression in older adults. Prior research has demonstrated that older adults are less likely than younger adults to endorse symptoms of dysphoria and sadness, and more likely to endorse appetite disturbance, anhedonia, and thoughts of death. Research has also demonstrated that clinicians are more likely to detect depression when it is more severe and when the patient has received prior mental health services. Additionally, several studies have demonstrated that clinicians often fail to recognize depression when the chief symptoms are somatic in nature. Though, prior research has not established whether clinicians may be more likely to diagnose depression based on the particular symptoms that are endorsed (i.e. depressed mood). Research 
on this topic may help to explain low rates of clinician detection of depression in late-life and may have implications for the assessment of depression in older adults.

\section{Specific Aim. Characterize the relation between depressive symptom endorsement and detection of depression in older adults.}

Based on prior research, it is hypothesized that greater depressive symptom endorsement will be associated with clinician detection of late-life depression (Simon \&VonKorff, 1995). Furthermore, because a number of factors have been identified that may cause clinicians to privilege sadness or depressed mood, it is hypothesized that clinicians will be more likely to detect depression in older adults if the patient endorses that particular symptom. In addition, because older adults with cognitive impairment are also less likely to endorse dysphoria and because of the difficulty in distinguishing between cognitive impairment and depression, presence of cognitive impairment is also hypothesized to be a factor that may interfere with the clinician detection of depression in late life.

Hypothesis 1: Greater depressive symptom endorsement will increase the likelihood that clinicians will diagnose late-life depression.

Hypothesis 2: Endorsement of depressed affect will moderate the relation between overall level of depressive symptoms and clinician detection of depression. Overall depressive symptoms will be more strongly related to the diagnosis of depression when symptoms of depressed affect are endorsed.

Hypothesis 3: Presence of cognitive impairment will moderate the relation between overall level of depressive symptoms and the diagnosis of depression in older adults. Specifically, overall depressive symptoms will be more strongly related to the diagnosis of depression in the absence of cognitive impairment. 
Method

\section{Participants}

Participants for the current study were obtained from the Swedish Twin Registry (STR). The registry was originally established in the early 1960's to study the effects of smoking on health (Cederlöf, 1966). The STR is now the largest well-characterized twin registry in the world, and, over the years, many research projects have been initiated using the registry. The current study utilized data collected from one such project known as the Swedish Adoption/Twin Study of Aging (SATSA; Pedersen, Plomin, Nesselroade, \& McClearn, 1992). SATSA data collection began with a comprehensive questionnaire in 1984 and subsequent questionnaires were administered in 1987 (Q2), 1990 (Q3), 1993 (Q4), and 2004 (Q5). Additionally, a subset of the twins was recruited to participate in a more comprehensive battery (including an evaluation of cognitive abilities) via in-person testing (IPT) at five time periods between 1985 and 2004. Only data collected between 1986 and 1994 were used for the current study, since the Center for Epidemiologic Studies- Depression Scale (CES-D) was not included in 1984 and medical diagnoses have not been coded through 2004.

A total of 3,838 individuals were originally contacted to participate in the SATSA project. Not all participants responded to the questionnaires at each time point, with 1,637 at Q2, 1,406 at Q3, and 1,450 at Q4. Considering the sample at the time of Q2, the response rates for those alive at Q3 and Q4 were 86\% and 90\% respectively (Wetherell, Gatz, \& Pedersen, 2001). A smaller subset of participants was included in the in-person testing with 645 individuals participating at IPT1 (1986-1988), 595 at IPT2 (1989-1991), and 569 at IPT3 (1992-1994). Medical records have been coded for a smaller subset of 476 individuals who participated in the in-person testing. Three hundred forty-four of these individuals were aged 50 or older at the 
time of Q2 in 1987. Because the current study focuses on the detection of late-life depression, only participants aged 50 and older at the time of this surveywere included. Thus, the final sample size for the present study was 344. Of these 344, there were 138 men (40.1\%) and 206 women $(59.9 \%)$. The average age of participants at the time of Q2 was $61.2(\mathrm{SD}=7.4)$.

The SATSA project was chosen for the present study because it includes a large, wellcharacterized, population-based sample with measures of depressive symptoms and cognitive functioning at several time points, in addition to coded primary care diagnoses.

\section{Measures}

Depressive Symptoms. Depressive symptoms were assessed by item endorsement on the Center for Epidemiologic Studies- Depression Scale (CES-D). The CES-D is a twenty-item measure that asks individuals to respond to items on a 4-pt Likert-type scale based on their symptomsin the past week. The CES-D contains items that assess depressed affect, well-being (or absence thereof), somatic complaints, psychomotor retardation, interpersonal difficulties, and other symptoms of depression. The CES-D has demonstrated good internal consistency, testretest reliability, and validity (Radloff, 1977), and has been shown to have measurement equivalence across the lifespan (Gatz, Johansson, Pederson, Berg, \& Reynolds, 1993). For the SATSA project, items were translated into Swedish and were backtranslated by another individual to ensure that the original meanings were retained. Factor analyses have shown evidence for four factors; depressed affect, psychomotor retardation, absence of well-being, and interpersonal difficulties (Gatz et al., 1993). This is a similar factor structure to that found in U.S. samples, suggesting that the measure functions in a similar way across these cultures. A cut-off score of 16 on the CES-D is the most commonly validated criterion (Craig \& Van Natta, 1973; Radloff, 1977). Furthermore, the majority of studies that utilize the CES-D have used 16 
as the cut-off score for clinically significant depressive symptoms, including those with older adult samples (e.g., Gatz \& Hurwicz, 1990). There is some debate with regard to whether the cutoff should be raised for older adults due to items relating to physical illness and bereavement (Beekman, Deeg, Limbeek, Braam, de Vries, \& van Tilberg, 1997). For example, one study found the CES-D to have high sensitivity (96.2\%), but poor specificity (23.8\%) using a cutoff of 16 in one study with Dutch older adults (ages 55-85) with or without MDD (Haringsma, Engels, Beekman, \& Spinhoven, 2004). However, the CES-D has been shown to have only minimal overlap with physical illness (Berkman et al., 1989).

Descriptive statistics and Pearson's correlations for the CES-D at each of the 6 time points (Q2, Q3, Q4, IPT1, IPT2, and IPT3) are presented in Table 1. Correlations between the CES-D at various time points ranged from .57-.74 (all p’s<.001). These significant correlations corroborate prior findings that CES-D scores are relatively stable over time (Fiske, Gatz, \& Pedersen, 2003). This pattern of stability also appeared to be true for those with higher CES-D scores. For example, for those above the clinical cutoff of 16 at the time of Q2, CES-D scores at Q2 were significantly related to CES-D scores at all five other time points (r’s=.28-.69, all p’s<.05). In the current study, the participant's highest CES-D at any time point was used for each analysis. The mean of participants' highest CES-D scores was $15.4(\mathrm{SD}=9.1)$. The "high CES-D” variable also met the underlying assumption of normality, with acceptable skew (.81) and kurtosis (.26).

Depressed Affect. Endorsement of depressed affect (sadness/dysphoria) was determined using two items from the CES-D. These two items were selected based on face validity and included "I felt depressed” and “I felt sad.” Other items on the “depressed affect” subscale were not included because they do not require the participant to endorse depressed mood. These items 
included "I thought my life had been a failure,” "I felt afraid,” “I felt lonely,” and "I cried.” As with the full scale CES-D, the participant's highest item endorsement of any time point was used for the current study. Descriptive statistics for these variables are presented in Table 2.

Cognitive Functioning. Cognitive abilities were determined using data from the cognitive battery included in the in-person testing (IPT) portion of SATSA. Subjects were administered tests of fluid intelligence, crystallized intelligence, and specific cognitive abilities. In particular, an overall measure of "general cognitive ability” was compiled using data from all the different subtests including block design, synonyms, information, figure logic, digit span, Thurstone's picture memory, names and faces (immediate and delayed), card rotations, digit symbols, analogies, and figure identification (see Table 3 for more detail). Specifically, this measure of "general cognitive ability" was created using the $1^{\text {st }}$ principal component of all the measures described residualized for gender. In the present study, the measure of cognitive ability was available for 317 of the 344 participants for at least one of the three IPTs. The lowest cognitive score at these three time points was used in the present analyses $(\mathrm{M}=-0.03, \mathrm{SD}=0.93$, skew $=0.00$, kurtosis $=0.15$ ). Lower scores on this overall measure are indicative of poorer cognitive functioning.

Clinician Detection of Depression. Clinician detection of depression was assessed using medical records coded by a project physician according to the ICD-10 diagnostic rubric. Relevant diagnoses included ICD F30-F34 (Depression) and ICD F32.0 (Depression, mild). The coded diagnoses for depression also included the year in which the diagnosis was given. Diagnoses were considered in the analyses if they were given between 1986 and 1994 . In the current study, 14 out 344 participants (4.1\%) were diagnosed with depression during 1986-1994. Seven participants were diagnosed with major depressive disorder and 7 participants were 
diagnosed with mild depression. Of those with a depression diagnosis, 4 were men (28.6\%) and 10 were women $(71.6 \%)$.

Analyses

For the present study, a series of simple logistic regressions were conducted. All of the independent variables (i.e., overall depressive symptoms, endorsement of depressed affect, and level of cognitive functioning) were tested as continuous variables. The rationale for this strategy was two-fold. (1) The use of continuous variables allowed for greater power than dichotomous variables. This was especially important in the present study, since the outcome variable (i.e., diagnosis of depression) was a relatively rare event, occurring in only $4.1 \%$ of participants. (2) By examining the variables as continuous measures, variability was preserved that would otherwise have been lost through the use of a dichotomy (i.e., not above clinical cutoff on the CES-D vs. above clinical cutoff on the CES-D). For example, a person with a CES-D score of 16 seemed qualitatively different from a person with a CES-D score of 40, even though both were above the clinical cutoff. Likewise, a person who rated the "I felt depressed" item as a 3 ("most or all of the time”) seemed more likely to endorse depressed affect to their physician compared to a person who rated the same item as a 1 ("some or little of the time”). Logistic regression makes no assumptions about the distribution of the independent variables; however, preliminary analyses assessed normality. All of the test variables had skew and kurtosis values between -1 and 1 , suggesting that none deviated significantly from normality. In all of the regression models, continuous variables were centered to avoid problems associated with multicolinearity. Furthermore, though the current study utilized a twin sample, it did not make use of the genetically informative nature of the dataset. Thus, corrections were made to confidence intervals using robust standard errors to adjust for non-independence of observations. 
In order to make these corrections, a SAS macro was employed (SAS Institute, 2003).

Specifically, this macro assessed the correlation between twin pairs and increased the variance estimates in proportion with the correlation. This expanded the confidence intervals and allowed for a more conservative estimate of the effect.

Results

Hypothesis 1: Greater level of depressive symptoms will increase the likelihood that clinicians will diagnose late-life depression.

For this hypothesis, the participant's highest CES-D score (at any of the time points included) was used as an indicator oflevel of depressive symptoms. A logistic regression analysis was conducted with highest score on the CES-D as the independent variable and diagnosis of depression as the dependent variable. After controlling for sex and age, CES-D scores were significantly related to clinician diagnosis, Wald Test $=12.61, \mathrm{p}<.001(\mathrm{OR}=1.10$, 95\% CI: 1.03-1.17). A one unit increase in scores on the CES-D increased the likelihood of receiving a diagnosis of depression by $10 \%$.

A follow-up analysis assessed the agreement between presence of clinically significant depressive symptoms (score $\geq 16$ on the CES-D at any time point) and clinician diagnosis of depression using a Kappa analysis. Overall, there appeared to be poor agreement between clinically significant scores on the CES-D and clinician diagnosis of depression (kappa $=.04$ ). One hundred forty-five participants (42.2\%) had a CES-D score greater than 16 at some point, compared to 14 (4.1\%) who received a depression diagnosis from a clinician.

Hypothesis 2: Endorsement of depressed affect will moderate the relation between overall level of depressive symptoms and clinician detection of depression. Greater level of 


\section{depressive symptoms will be more strongly related to the diagnosis of depression when depressed affect is endorsed.}

To test hypothesis 2, a logistic regression analysis was run with level of depressive symptoms and endorsement of depressed affect as predictors of clinician diagnosis of depression. An interaction term (overall level of depressive symptoms X endorsement of depressed affect) was then added to the model to determine whether endorsement of depressed affect moderated the relation between overall level of depressive symptoms and clinician diagnosis. Participants’ highest CES-D score (at any of the time points included) was used as an indicator of level of depressive symptoms. Likewise, participants’ highest rating of depressed affect items on the CES-D (“I felt depressed” plus “I felt sad”) was used as an indicator of endorsement of depressed affect. When both variables were present in the regression model, only the endorsement of depressed affect was significantly related to the clinician diagnosis of depression, Wald Test $=4.07, \mathrm{p}<.05$ (OR $=2.05$, 95\% CI: 1.42-4.10). Overall level of depressive symptoms no longer predicted clinician diagnosis, Wald Test $=0.00, \mathrm{p}=.96(\mathrm{OR}=$ 1.00, 95\% CI: 0.91-1.11). The interaction between overall level of depressive symptoms and endorsement of depressed affect approached, but did not reach, significance, Wald Test $=3.27$, p

$=.07(\mathrm{OR}=0.97,95 \% \mathrm{CI}: 0.94-1.03)$.

Logistic regression analyses were then run separately for the two items related to depressed affect, to examine whether the two items may perform differently. First, participants’ highest individual rating of the "sad" item was used instead of the combined depressed affect items. Unlike depressed affect, the endorsement of sadness was not related to the clinician diagnosis of depression independently of overall level of depressive symptoms, Wald Test $=$ 0.18, $\mathrm{p}=.67(\mathrm{OR}=1.26,95 \% \mathrm{CI}: 0.44-3.62)$. In addition, the endorsement of sadness did not 
moderate the relation between overall level of depressive symptoms and clinician diagnosis, Wald Test $=0.04, \mathrm{p}=.84(\mathrm{OR}=1.01,95 \% \mathrm{CI}: 0.94-1.07)$

Next, the participant's highest rating of the “depressed” item was examined. With both endorsement of the depressed item and overall level of depressive symptoms in the regression equation, only the endorsement of the depressed item was related to the clinician diagnosis of depression, Wald Test $=7.39, \mathrm{p}<.01(\mathrm{OR}=2.75,95 \% \mathrm{CI}: 1.33-5.69)$. Overall level of depressive symptoms was no longer independently related to clinician diagnosis after accounting for endorsement of the depressed item, Wald Test $=0.54, \mathrm{p}=.46(\mathrm{OR}=1.03,95 \% \mathrm{CI}$ : 0.961.10). The interaction between overall level of depressive symptoms and endorsement of the depressed item was also significant, Wald Test $=5.37, \mathrm{p}<.05(\mathrm{OR}=1.07,95 \% \mathrm{CI}: 1.02-1.12)$, indicating that the endorsement of the depressed item moderated the relation between overall level of depressive symptoms and the clinician diagnosis of depression. To further characterize this relation, separate regression analyses were run examining the relation between overall level of depressive symptoms in those with higher endorsement of the depressed item versus those with lower endorsement of depression utilizing a mean split. As anticipated, the relation between overall level of depressive symptoms and clinician diagnosis of depression was stronger in those with higher endorsement on the depressed item, Wald Test $=8.85, \mathrm{p}<.01(\mathrm{OR}=1.11$, 95\% CI: 1.04-1.19). The relation between overall symptoms and depression diagnosis was not significant in those with lower endorsement on the depressed item, Wald Test $=0.24, \mathrm{p}=.62$ $(\mathrm{OR}=1.07,95 \% \mathrm{CI}:$ 0.83-1.37). None of the relations described was altered after including sex and age as covariates.

Hypothesis 3: Cognitive functioning will moderate the relation between overall level of depression and diagnosis of depression in older adults. Specifically, clinically significant 


\section{depressive symptoms will be more strongly related to the diagnosis of depression in the absence of cognitive impairment.}

In order to test hypothesis 3, a logistic regression analysis was run with overall level of depressive symptoms and cognitive functioning as predictors of the clinician diagnosis of depression. An interaction term (overall level of depressive symptoms X cognitive functioning) was then added to the model to determine whether cognitive functioning moderated the relation between overall level of depressive symptoms and clinician diagnosis. Because the presence/absence of cognitive impairment was the target variable, participants’ lowest “general cognitive ability” score was used as an indicator of cognitive functioning. Cognitive functioning was not related to depression diagnosis over and above overall symptoms of depression, Wald Test $=0.21, \mathrm{p}=.65(\mathrm{OR}=1.17,95 \% \mathrm{CI}: 0.59-2.23)$. In addition, the inclusion of the interaction between overall level of depressive symptoms and cognitive functioning did not add anything to the model, Wald Test $=0.68, \mathrm{p}=.41(\mathrm{OR}=0.97,95 \% \mathrm{CI}: 0.89-1.05)$. A post-hoc descriptive analysis revealed that mean cognitive functioning scores were slightly lower for individuals with significant self-reported depressive symptoms (i.e., CES-D $\geq 16$ ), but no clinician diagnosis of depression $(n=128, M=-0.30, S D=0.88)$ compared to those with significant self-reported diagnosis and a clinician diagnosis of depression $(n=7, M=-0.25$, $S D=0.60)$. However, this difference was not statistically significant.

\section{Discussion}

Findings from the present study affirm that symptom presentation plays an important role in the clinician assessment of late-life depression. Results suggest that overall level of depressive symptoms is related to receiving a clinician diagnosis of depression. In addition, this relationship is moderated by the endorsement of a single item on the Center for Epidemiologic 
Studies- Depression Scale (i.e., “I felt depressed”). When this particular item is rated highly, the relation between overall level of depressive symptoms and clinician diagnosis of depression is stronger compared to lower ratings of the same item. However, other items on the CES-D related to depressed affect (i.e., "I felt sad”) do not appear to have the same moderating affect. These findings suggest that clinicians are particularly focused on the endorsement of “depression” versus the endorsement of depressed affect more generally in older adults. This relationship is especially pertinent to older adults, given that this population is less likely to endorse depressed affect and less likely to use the term “depression” compared with younger adults (Gallo \& Rabbins, 1999). The notion that clinicians are biased toward detecting depression only in the context of endorsement of the "depression” item may lead to false negatives in the diagnosis of depression in late life.

Findings from the current study also corroborate prior studies that show a disparity between level of depressive symptoms in late-life and prevalence of diagnoses. Forty-two percent of older adults in the present sample had clinically relevant levels of depressive symptoms on the CES-D, but only $4 \%$ had been diagnosed with depression by a clinician. A number of factors may play a role in this relation. For example, it has been hypothesized that older adults' scores on the CES-D may be inflated due to higher rates of physical illness and bereavement (Beekman et al., 1997). However, it is also possible that ageism may be responsible for the misattribution of somatic symptoms to aging as opposed to symptoms of depression.

Unlike the endorsement of the depression item, the present study shows that level of cognitive functioning does not moderate the relation between overall level of depressive symptoms and clinician diagnosis of depression. There are a number of reasons that may help 
explain this non-finding. All of the participants in the present sample were able to complete selfreported measures of depression, suggesting that more severe levels of cognitive impairment may not have been represented (i.e., moderate-severe dementia of the Alzheimer's type). It is also possible that there has been an increase in the awareness of the high prevalence of depression in the context of dementia. For example, those suspected of dementia may have undergone a more thorough medical or neuropsychological evaluation, which often includes formal screening for depression.

Though the prevalence of major depression declines with age, endorsement of depressive symptomatology increases in older adults. In addition, older adults suffering from depression are at a higher risk than non-depressed older adults for functional impairment, cognitive impairment, social impairment, death, and suicide. These risk factors make the accurate and valid assessment of depression in late life a critical public health issue. This study examined the idea that clinicians may be differentially biased in diagnosing depression if sadness/dysphoria is endorsed. This study also examined whether presence of cognitive impairment may be a barrier to the diagnosis of depression in late life. These are especially pertinent to older adults, as older adults are more likely than younger adults to have either cognitive impairment or non-dysphoric depression. As identified in the study, the privileged symptom of endorsement of "depression" may result in the under-diagnosis of depression in late life.

There are a few limitations in the present study. For one, the study operationally defines clinician detection of depression as assigning a diagnosis of major or minor depression. It is possible that clinicians may detect depression without giving a diagnosis (Rost, Smith, Matthews, \& Guise, 1994). This phenomenon could inflate the findings of the current project. Though, if a diagnosis for a patient is not rendered, it is unlikely that they are receiving treatment 
for depression. Another potential limitation is that the present study operationally defines endorsement of depressed affect by item endorsement on the CES-D. However, we do not know what information the participants disclose to their physicians regarding depressive symptom endorsement. Nevertheless, we found a significant relation between self-reported depressive symptoms and clinician diagnosis of depression in the present study. Another potential limitation is that the timing of CES-D administration and physician office visits was not coordinated. Though, it should be noted that CES-D scores are quite stable over time (Fiske et al., 2003). These limitations notwithstanding, the present study was the first to examine clinician bias toward diagnosing depression when depressed mood is endorsed.

Regarding the clinical implications of the present study, results suggest that clinicians may benefit from improved awareness of the unique presentation of depression in late life. Clinicians who are differentially biased in diagnosing depression in the presence of endorsement of feeling “depressed” are likely to miss many depressed older adults who do not use this language or who present with predominant psychomotor symptoms of depression. Thus, programs that educate clinicians about the unique aspects of late-life depression may aid in correcting this bias.

It should be noted that older adults benefit from many of the same psychotherapy interventions as younger adults, including individual, group, and family therapies (Karel \& Hinrichsen, 2000). A number of evidence-based treatments exist for depression in late life, including cognitive behavioral therapies (e.g., Coon, Rider, Gallagher-Thompson, \& Thompson, 1999), interpersonal psychotherapies (e.g., Hinrichsen, 1999), and some pharmacological treatments (see Beyer, 2007). A number of psychosocial treatments for depression have also been shown to be effective in older adults with dementia (see Teri, McKenzie, \& LaFazia, 2005). 
However, it has been noted that psychological interventions for depression are underutilized in older adults compared to pharmacological treatments for depression (Fiske, Wetherell, \& Gatz, 2009). With improved detection of depression in late life, these therapies may be available to more individuals who could really benefit from treatment for depression. 


\section{References}

American Psychiatric Association. (2000). Diagnostic and Statistical Manual of Mental Disorders-Fourth Edition-Text Revision. Washington, DC: Author.

Alexopoulos, G. S., Borson, S., Cuthbert, B. N., Devanand, D. P., Mulsant, B. H., Olin, J. T., et al. (2002).Assessment of late life depression. Biological Psychiatry, 52, 164-174.

Bédard, M., Molloy, D. W., Squire, L., Minthorn-Biggs, M. B., Dubois, S., Lever, J. A., et al. (2003). Validity of self-reports in dementia research: The Geriatric Depression Scale. Clinical Gerontologist, 26, 155-162.

Beekman, A. T.,DeegD. J., Limbeek A., Braam, A. W., de Vries, M. Z.,\& van Tilberg, W. (1997). Criterion validity of the Center for Epidemiologic Studies Depression scale (CES-D): Results from a community based sample of older adults in the Netherlands. Psychological Medicine, 27, 231-235.

Berkman, L. F., Berkman, C.S., Kasl, S., Freeman, D. H. Jr., Leo, L., Ostfeld, A. M. \& et al. (1986). Depressive symptoms in relation tophysical health and functioning in the elderly. American Journal of Epidemiology, 124, 372-388.

Beyer, J. L. (2007).Managing depression in geriatric populations.Annals of Clinical Psychiatry, 19, 221-228.

Blazer, D. G. (2003). Depression in late life: Review and commentary. Journal of Gerontology A: Biological Sciences \& Medical Sciences, 58, 249-265.

Bogner, H. R., de Vries, H. F., Maulik, P. K., \&Unützer, J. (2009). Mental health services use: Baltimore Epidemiologic Catchment Area follow-up. American Journal of Geriatric Psychiatry, 17, 706-715.

Cederlöf, R. (1966). The twin method in epidemiological studies on chronic disease. Stockholm, 
Sweden: Karolinska Institutet.

Conwell, Y., \& Brent, D. (1996). Suicide and aging I: Patterns of psychiatric diagnosis. In Suicide: International Perspectives, ed. J. L. Pearson, Y. Conwell, pp. 15-30. New York: Springer.

Coon, D. W., Rider, K., Gallagher-Thompson, D., \& Thompson, L. (1999). Cognitive-behavioral therapy for the treatment of late-life distress. In M. Duffy (ed.), Handbook of counseling and psychotherapy with older adults (pp. 487-510). New York: John Wiley \& Sons.

Coyne, J. C., Schwenk, T. L., \& Fechner-Banks, S. (1995). Nondetection of depression by primary care physicians reconsidered. General Hospital Psychiatry, 17, 3-12.

Craig, T. J., \& Van Natta, P. A. (1973). Validation of the Community Mental Health Assessment Interview instrument among psychiatric inpatients. Hagerstown, MD. Community Mental Health Assessment Program.

Djernes, J. K. (2006). Prevalence and predictors of depression in populations of the elderly: A review. ActaPsychiatricaScandinavica, 113, 372-387.

Docherty, J. P. (1997). Barriers to the diagnosis of depression in primary care.Journal of Clinical Psychiatry, 58(Suppl 1), 5-10.

Edelstein, B. A., Drozdick, L. W., \& Ciliberti, C. M. (2010). Assessment of depression and bereavement in older adults. In P. A. Lichtenberg (Ed.), Handbook of Assessment in Clinical Gerontology (pp. 3-44). New York, NY: John Wiley \& Sons.

Ehrt, U., Brønnick, K., Leentjens, A. F., Larsen, J. P., \& Aarsland, D. (2006). Depressive symptom profile in Parkinson's disease: A comparison with depression in elderly patients without Parkinson's disease. International Journal of Geriatric Psychiatry, 21, 252-258.

Fiske, A., Gatz, M., \& Pedersen, N. (2003). Depressive symptoms and aging: The effects of 
illness and non-health-related events. The Journals of Gerontology: Psychological Sciences, 58(6), 320-328.

Fiske, A., \& O’Riley (2008). Depression in late life. In J. Hunsley \& E. J. Marsh (Eds.), A Guide to Assessments that Work. New York: Oxford University Press.

Fiske, A. E., Wetherell, J. L., \& Gatz, M. (2009).Depression in older adults.Annual Review of Clinical Psychology, 5, 363-389.

Gallo, J. J., Anthony, J. C., \& Muthén, B. O. (1994). Age differences in the symptoms of depression: A latent trait analysis. Journal of Gerontology, 49(6), 251-264.

Gallo, J. J. \& Rabins, P. V. (1999). Depression without sadness: Alternative presentations of depression in late life. American Family Physician, 60, 820-826.

Gallo, J. J., Rabins, P. V., Lyketsos, C. G., Tien, A. Y., \& Anthony, J. C. (1997). Depression without sadness: Functional outcomes of nondysphoric depression in later life. Journal of the American Geriatrics Society, 45, 570-578.

Gallo, J. J., Ryan, S. D., \& Ford, D. E. (1999). Attitudes, knowledge, and behavior of family physicians regarding depression in late life. Archives of Family Medicine, 8, 249-256.

Gatz, M., \& Hurwicz, M. (1990). Are old people more depressed? Cross-sectional data on Center for Epidemiological Studies Depression Scale factors. Psychology and Aging, 5(2), 284-290.

Gatz, M., Johansson, B., Pedersen, N. L., Berg, S., \& Reynolds, C. (1993). A cross-national selfreport measure of depressive symptomatology. International Psychogeriatrics, 5, 147156.

Haringsma, R., Engels, G. I., Beekman, A. T. F., \& Spinhoven, P. (2004). The criterion validity of the Center for Epidemiological Studies Depression Scale (CES-D) in a sample of self- 
referred elders with depressive symptomatology. International Journal of Geriatric Psychiatry, 19, 558-563.

Harman, J. S., Veazie, P. J., Lyness, J.M. (2006). Primary care physician office visits for depression by older Americans. Journal of General Internal Medicine,21, 926-930.

Hasin, D. S., Goodwin, R. D., Stinson, F. S., \& Grant, B. F. (2005). Epidemiology of major depressive disorder: Results from the National Epidemiologic Survey on Alcoholism and Related Conditions. Archive of General Psychiatry, 62, 1096-1106.

Hinrichsen, G. A. (1999). Interpersonal psychotherapy for late-life depression. In M. Duffy (ed.), Handbook of counseling and psychotherapy with older adults (pp. 470-486). New York: John Wiley \& Sons.

Jeste, D. V., Blazer, D. G., \& First, M. (2005). Aging-related diagnostic variations: Need for diagnostic criteria appropriate for elderly psychiatric patients. Biological Psychiatry, 58, $265-271$.

Jha, A., Tabet, N., \&Orrell, M. (2001). To tell or not to tell- comparison of older patients’ reaction to their diagnosis of dementia and depression. International Journal of Geriatric Psychiatry, 16, 879-885.

Katon, W., \&Schulberg, H. (1992).Epidemiology of depression in primary care.General Hospital Psychiatry, 14, 237-247.

Karel, M. J., \&Hinrichsen, G. (2000). Treatment of depression in late life: Psychotherapeutic interventions. Clinical Psychology Review, 17, 847-879.

Klinkman, M. S. (2003). The role of algorithms in the detection and treatment of depression in primary care. The Journal of Clinical Psychiatry, 64(suppl 2), 19-23.

Klinkman, M. S., Coyne, J. C., Gallo, S. \&Schwenk, T. L. (1998). False positives, false 
negatives, and the validity of the diagnosis of major depression in primary care. Archives of Family Medicine, 7, 451-461.

Klinkman, M. S., Schwenk, T. L., \& Coyne, J. C. (1997).Depression in primary care- more like asthma than appendicitis: the Michigan Depression Project. Canadian Journal of Psychiatry, 42, 966-973.

Licht-Strunk, E., Beekman, A., de Haan, M., \& van Marwijk, H. (2009). The prognosis of undetected depression in older general practice patients. A one year follow-up study. Journal of Affective Disorders, 114(1-3), 310-315.

Licht-Strunk, E., van der Kooij, K., van Schaik, D., van Marwijk, H., van Hout, H., de Haan, M., et al. (2005). Prevalence of depression in older patients consulting their general practitioner in The Netherlands. International Journal of Geriatric Psychiatry, 20(11), 1013-1019.

Logsdon, R. G., McCurry, S. M., \& Teri, L. (2008). Assessment and treatment of dementiarelated affective disturbances. Handbook of emotional disorders in later life: Assessment and treatment (pp. 345-362). New York, NY: Oxford University Press.

Lopez, O. T., Becker, J. T., Sweet, R. A., Klunk, W., Kaufer, D. I., Saxton, J., \& et al. (2003). Psychiatric symptoms vary with the severity of dementia in probable Alzheimer's disease. Journal of Neuropsychiatry and Clinical Neuroscience, 15(3), 346-353.

Lyness, S. A., Zarrow, C., \& Chui, H. C. (2003). Neuron loss in key cholinergic and aminergic nuclei in Alzheimer's disease: A meta-analysis. Neurobiology of Aging, 24, 1-23.

Mehta, K. M., Yaffe, K., Langa, K., Sands, L., Whooley, M., \&Covinsky, K. (2003). Additive effects of cognitive function and depressive symptoms on mortality in elderly community-living adults. The Journals of Gerontology: Series A: Biological Sciences 
and Medical Sciences, 58A(5), 461-467.

Menchetti, M., Murri, M., Bertakis, K., Bortolotti, B., \&Berardi, D. (2009). Recognition and treatment of depression in primary care: Effect of patients' presentation and frequency of consultation. Journal of Psychosomatic Research, 66(4), 335-341.

Mitchell, A. J., Vaze, A., \&Rao, S. (2009). Clinical detection of depression in primary care: A meta-analysis.The Lancet, 334, 609-619.

Newmann, J. P. (1989). Aging and depression.Psychology of Aging, 4, 150-165.

Newmann, J. P., Engel, R. J., \& Jensen, J. E. (1991). Age differences in depressive symptom experiences. Journal of Gerontology, 46, P224-P235.

Olin, J. T., Katz, I. R., Meyers, B. S., Schneider, L. S., \&Lebowitz, B. D. (2002). Provisional diagnostic criteria for Depression of Alzheimer Disease: Rationale and background. American Journal of Geriatric Psychiatry, 10, 129-141.

Paradiso, S., Vaidya, J., Tranel, D., Kosier, T., \& Robinson, R. G. (2008).Nondyshphoric depression following stroke.Neuropsychiatry and Clinical Neuroscience, 20, 52-61.

Park, J. H., Lee, S. B., Lee, T. J., Lee, D. Y., Jhoo, J. H., \& et al. (2007). Depression in vascular dementia is quantitatively and qualitatively different from depression in Alzheimer's disease. Dementia and Geriatric Cognitive Disorders, 23, 67-73.

Pedersen, N. L., Plomin, R., Nesselroade, J. R., \&McClearn, G. E. (1992).A quantitative genetic analysis of cognitive abilities during the second half of the life span.Psychological Science, 6, 346-353.

Radloff, L. S. (1977). The CES-D Scale: A self-report depression scale for research in the general population. Applied Psychological Measurement, 1, 385-401. 
Rothermund, K., \&Meiniger, C. (2004). Stress-buffering effects of self-complexity: Reduced affective spillover or self-regulatory processes? Self and Identity, 3(3), 263-281.

Rost, K., Smith, G. R., Matthews, D. B., \& Guise, B. (1994).The deliberate misdiagnosis of major depression in primary care.Archives of Family Medicine, 3(4), 333-337.

SAS Institute. (2003). SAS system for Microsoft Windows, version 9 (Computer software). Cary, NC. SAS Institute Inc.

Schweitzer, I., Tuckwell, V., O’Brien, J., \& Ames, D. (2002). Is late onset depression a prodrome to dementia? The International Journal of Geriatric Psychiatry, 17, 997-1005.

Simon, G. E., \&VonKorff, M. (1995).Recognition, management, and outcomes of depression in primary care.Archives of Family Medicine, 4(2), 99-105.

Steffens, D. C., \& Potter, G. C. (2007). Geriatric depression and cognitive impairment. Psychological Medicine, 38, 163-175.

Storandt, M., \&Vandenbos, G. (1994).Neuropsychological Assessment of Dementia and Depression in Older Adults: A Clinician's Guide. Washington, D.C.: American Psychological Association.

Teri, L., McKenzie, G., \&LaFazia, D. (2005). Psychosocial treatment of depression in older adults with dementia. Clinical Psychology: Science and Practice, 12(3), 303-316.

U.S. Preventive Services Task Force. (2009). Screening for depression in adults: U.S. Preventive Services Task Force recommendation statement. Internal Medicine, 151(11), 784-792.

Watson, L. C., Lewis, C. L., Kistler, C. E., Amick, H. R., \&Boustani, M. (2004). Can we trust depression screening instruments in healthy 'old-old’ adults? International Journal of Geriatric Psychiatry, 19, 278-285. 
Wetherell, J. L., Gatz, M., and Pedersen, N. L. (2001). A longitudinal analysis of anxiety and depressive symptoms. Psychology and Aging, 16(2), 187-195. 
Table 1. Means, standard deviations, and Pearson's correlations for Center for Epidemiologic Studies Depression Scale at each time point.

\begin{tabular}{lcccccccc}
\hline Time Point & $\mathrm{n}$ & Mean & SD & Q3 & Q4 & IPT1 & IPT2 & IPT3 \\
\hline Q2 & 314 & 9.9 & 7.9 & .67 & .59 & .74 & .61 & .61 \\
Q3 & 317 & 10.6 & 7.8 & - & .63 & .57 & .67 & .64 \\
Q4 & 323 & 11.5 & 8.9 & - & - & .62 & .63 & .64 \\
IPT1 & 111 & 11.1 & 8.5 & - & - & - & .57 & .60 \\
IPT2 & 274 & 9.7 & 8.1 & - & - & - & - & .64 \\
IPT3 & 260 & 9.4 & 6.7 & - & - & - & - & -
\end{tabular}

Note: Q= Questionnaire, IPT= In-Person Testing. All correlations significant at the $\mathrm{p}<.001$ level. 
Table 2. Descriptive statistics for participant's highest Center for Epidemiologic StudiesDepression scale (CES-D), depressed affect total, and individual item totals at any time point.

\begin{tabular}{lccccc}
\hline Scale/Item & $\mathrm{n}$ & Mean & SD & Skewness & Kurtosis \\
\hline Total CES-D & 344 & 15.44 & 9.13 & 0.81 & 0.26 \\
Depressed Affect Total & 344 & 1.71 & 1.47 & 0.62 & -0.46 \\
'Depressed' Item & 344 & 0.82 & 0.90 & 0.90 & -0.06 \\
'Sad' Item & 344 & 1.00 & 0.81 & 0.46 & -0.34 \\
\end{tabular}


Table 3. Measures of Cognitive Ability from SATSA ${ }^{\mathrm{a}}$

\begin{tabular}{|c|c|c|}
\hline Test Name & $\begin{array}{c}\text { Domain/Specific Cognitive } \\
\text { Ability }\end{array}$ & Resource \\
\hline $\begin{array}{l}\text { Digit Span Forward and } \\
\text { Backward }\end{array}$ & Memory & WAIS (Swedish version) \\
\hline $\begin{array}{l}\text { Thurstone’s Picture } \\
\text { Memory }\end{array}$ & Memory & DS Battery \\
\hline $\begin{array}{l}\text { Names and Faces } \\
\text { (Immediate \& Delayed) }\end{array}$ & Memory & Colorado Adoption Project \\
\hline Information & Crystallized/Verbal & WAIS (Swedish version) \\
\hline Synonyms & Crystallized/Verbal & DS Battery \\
\hline Analogies & $\begin{array}{l}\text { Fluid and crystallized/Verbal, } \\
\text { reasoning }\end{array}$ & WIT-III \\
\hline Figure Logic & Fluid/Spatial, reasoning & DS Battery \\
\hline Kohs Block Design & Fluid/Spatial & DS Battery \\
\hline Card Rotations & Spatial & Educational Testing Service \\
\hline Digit Symbol & Perceptual speed & WAIS \\
\hline Figure Identification & Perceptual speed & DS Battery \\
\hline
\end{tabular}

Note: ${ }^{\mathrm{a}}$ Table adapted from Pedersen, Plomin, Nesselroade, \& McClearn, 1992; WAIS = Wechsler Adult Intelligence Scale; DS Battery = Dureman-Salde Battery. 